\title{
Effects of food deprivation on learning and expression of flavor preferences conditioned by saccharin or sucrose
}

\author{
ELIZABETH D. CAPALDI, JACQUELINE OWENS, and KATHERINE A. PALMER \\ University of Florida, Gainesuille, Florida
}

\begin{abstract}
In four experiments, food deprivation was varied during conditioning and testing of conditioning of flavor preferences by sweeteners. Conditioned preferences for a flavor associated with a more concentrated solution were enhanced by increased deprivation in training whether sucrose or saccharin was used when rats consumed solutions freely during training. When consumption of solutions was controlled and higher deprivation levels were used, preference for the higher concentration of sucrose was still enhanced by increased deprivation in training, but this did not occur with saccharin. We suggest that deprivation may enhance the reinforcing value of sweetness only when calories increase along with sweetness. We also suggest that deprivation can enhance flavor preference learning by increasing consumption and thereby increasing exposure to the flavored solutions.
\end{abstract}

Incentive theories of deprivation effects assume that deprivation increases the reward value of a goal (Bolles, 1972). One way of testing this idea is to give rats one food under high deprivation and another under low deprivation. If deprivation affects the reward value of food and affects the reinforcement produced by that food, rats should later prefer the food they have had under high food deprivation to the one they have had under low deprivation.

In an early experiment of this type, Revusky (1967) gave grape juice or milk before or after a meal; thus one food was given under high deprivation, before feeding, and the other food was given under low deprivation, after feeding. The rats later showed increased preference for the flavor given under high deprivation-before feeding. However, when flavors are given immediately before and after meals, factors other than deprivation level can affect preference. The high-deprivation flavor precedes the meal and preference can increase for this flavor because of its association with the following meal. The lowdeprivation flavor follows the meal and preference for this flavor can change because of association with the postingestive effects of the meal.

In a series of experiments, Capaldi and Myers (1982) and Capaldi, Myers, Campbell, and Sheffer (1983) gave flavors under high and low deprivation either separately from meals, or, as Revusky (1967) did, before and after the daily feeding. Rats preferred the flavor given under high deprivation when this flavor also preceded the daily

\footnotetext{
This research was supported in part by Grant MH 39453 to the first author from the National Institute of Mental Health. Requests for reprints should be sent to E. D. Capaldi, Department of Psychology, University of Florida, Gainesville, FL 32611-2065.
}

meal, but preferred the flavor given under low deprivation when flavors were given separately from meals. In these studies, saccharin or sucrose solutions were used to deliver flavors. Capaldi, Sheffer, and Owens (1991) showed (again being careful to give flavors separately from meals) that these results generalized to sweet foods: if flavors were given in sweetened mash, rats preferred the flavor given under low deprivation, just as they do if flavors are given in saccharin or sucrose solutions. However, Capaldi et al. (1991) found that if flavors were given in unsweetened mash, rats preferred the flavor received under high deprivation to that received under low deprivation, which is consistent with incentive theories of deprivation. Capaldi et al. (1991) suggested that the reinforcing value of food is increased by deprivation; this is consistent with incentive theories of deprivation. However, sweetness is apparently less reinforcing under high deprivation than under low deprivation, so the use of sweet food reduces or eliminates the facilitating effect of deprivation on reward value.

The conclusion that sweetness is less reinforcing with increased deprivation seems paradoxical because increasing deprivation increases consumption of both sugar and saccharin (see, e.g., Smith \& Duffy, 1957). However, the studies by Capaldi and her colleagues did not measure deprivation's effects on current consumption, but rather measured deprivation's effects on learning produced by foods and liquids. Deprivation may enhance the current consumption of sweet substances, yet not necessarily increase the reinforcement produced by these substances.

The facilitating effects of deprivation on consumption of sweet substances may reflect an enhancement of the oral effects of sweet substances on ingestion (Davis \& Levine, 1977). Alternatively, the immediate effects of dep- 
rivation on consumption may reflect a previously learned or genetically mediated association of sweetness with calories. Fedorchak and Bolles (1987) showed that deprivation enhanced consumption of flavors associated with calories. Thus deprivation should enhance consumption of sweet substances if sweetness is associated with calories.

The purpose of the present experiments was to determine how deprivation affects learning of flavor preferences produced by sweeteners. That is, we examined the reinforcing effect of increased sweetness as a function of deprivation. Because increasing deprivation increases consumption of sweet substances, deprivation may affect the reinforcing effect of sweet substances merely by increasing exposure to those substances. That is, because a hungrier rat consumes more of a sweet substance, it may obtain more reinforcement from that substance. In Experiments 1 and 2 , we allowed consumption to vary freely and paired flavors with different concentrations of sucrose (Experiment 1) or with different concentrations of saccharin (Experiment 2). These experiments evaluated whether deprivation facilitated conditioning of flavor preferences by sweeteners when effects of deprivation on consumption were allowed to influence results.

In Experiments 3 and 4, we equated consumption under the different deprivation levels. If the effect of deprivation on conditioning is produced by deprivation's effect on consumption, there should be no effect of deprivation on conditioning when consumption under the different deprivation levels is equated. However, if deprivation enhances the oral effects of sweet substances or increases the value of calories associated with sweetness, preference for a flavor associated with greater sweetness should be enhanced by increased deprivation during conditioning even when consumption is equated under the different deprivation levels. In addition, if deprivation enhances the reinforcing effect of calories and the association between sweetness and calories is learned, deprivation will enhance learning of conditioned flavor preferences for a sweeter substance only if more calories are associated with this substance. This implies that deprivation should facilitate learning of conditioned flavor preferences when sucrose is used (Experiment 3), but not when saccharin is used (Experiment 4).

\section{EXPERIMENT 1}

\section{Method}

Subjects. The subjects were 41 naive Sprague-Dawley rats (Harlan, Indianapolis), 75 days old on arrival and 82 days old at the start of deprivation.

Materials. Flavored solutions were presented in 50-ml Nalgene centrifuge tubes with rubber stoppers and metal spouts. Flavor extracts were made of $2 \%$ imitation cinnamon or oil of wintergreen dissolved in ethanol. The sucrose solutions contained $1 \%$ or $8 \%$ sucrose and $1 \%$ cinnamon or wintergreen flavor extract dissolved in water. All measurements were volume/volume. Capaldi et al. (1983) used the same materials in their experiments.

Procedure. Water was available ad lib throughout the experiment, in clear plastic bottles mounted to the right of the food hop- per on the cage fronts. The rats were housed individually. All rations were fed in the cages, and all training and testing occurred in the rats' home cages. On each of 3 days prior to the start of the experiment, rats were weighed and the mean weight over these days was used to assign subjects to four groups matched on weight. The design was a $2 \times 2$ factorial combining deprivation level in training with deprivation level in testing. The groups' names refer to deprivation level ( $h i$ for high and $l o$ for low) in training and the test. There were 11 subjects in Group lo-lo and 10 subjects in each of the other groups. High deprivation consisted of a 14-g-per-day schedule. Rats were fed less than this as was needed to compensate for the calories consumed in sucrose; low deprivation consisted of ad-lib access to rat chow. The subjects' weights were monitored throughout the experiment. Training began 11 days after the start of deprivation. The tubes containing solutions were attached with wire springs to the front of the home cage, to the left of the water bottle.

On the 2 days prior to training, the rats were preexposed to flavored $8 \%$ sucrose and $1 \%$ sucrose for $24 \mathrm{~h}$ each $(40 \mathrm{ml})$, with half the rats having $8 \%$ sucrose on the day before $1 \%$ sucrose and half having the reverse order. In training, each subject received two tubes of solution each day for $15 \mathrm{~min}$, separated by a 1-h interval. One tube contained $8 \%$ sucrose; the other, $1 \%$ sucrose. For half the subjects in each group, cinnamon was paired with $8 \%$ sucrose and wintergreen with $1 \%$ sucrose; for the other half of the subjects, the flavor-solution pairings were reversed. On half the days, $8 \%$ sucrose preceded $1 \%$ sucrose; on the other half of the days, $1 \%$ sucrose preceded $8 \%$ sucrose. There were 10 days of training.

Following training, Group hi-lo was placed on chow ad lib and Group lo-hi was placed on the 14-g-per-day deprivation schedule. Groups lo-lo and hi-hi continued their training deprivation schedules. Testing began when weights had stabilized, 17 days after training ended. There were 2 test days, each involving a 10-min choice between cinnamon- and wintergreen-flavored solution. The two bottles in testing were placed to the left and right of the position that had contained the single bottle in training. For half the subjects, cinnamon and wintergreen were given in $8 \%$ sucrose on Day 1 of test and in 1\% sucrose on Day 2 of test; the other half of the subjects had flavors in $1 \%$ sucrose on Day 1 and $8 \%$ sucrose on Day 2. The positions of the bottle containing cinnamon and that containing wintergreen were reversed between tests. On each test, the tubes were inserted simultaneously in the grids adjacent to the training grid. When one solution was sampled, that spout was withdrawn. When the alternate solution was sampled, that spout was withdrawn for $1 \mathrm{sec}$ before both spouts were reinserted.

\section{Results}

In training, rats trained under high deprivation consumed a mean of $24.4 \mathrm{ml}$ of $8 \%$ sucrose and $3.0 \mathrm{ml}$ of $1 \%$ sucrose; the comparable figures for rats trained under low deprivation were $11.2 \mathrm{ml}$ and $2.1 \mathrm{ml}$. Analysis of variance showed that the difference due to deprivation was significant $[F(1,37)=108.02]$ (for this and all statistical tests an alpha level of .05 was used), as was the difference due to concentration of sucrose $[F(1,37)=801.29]$. The difference in consumption between $8 \%$ and $1 \%$ sucrose was larger for rats trained under high deprivation $[F(1,37)=130.6]$.

Figure 1 shows the results in testing. Shown are the mean milliliters consumed of the flavor that had been paired with $8 \%$ sucrose in training and the flavor that had been paired with $1 \%$ sucrose in training for each group. As can be seen in Figure 1, although all groups consumed more of the flavor that had been paired with $8 \%$ sucrose, 


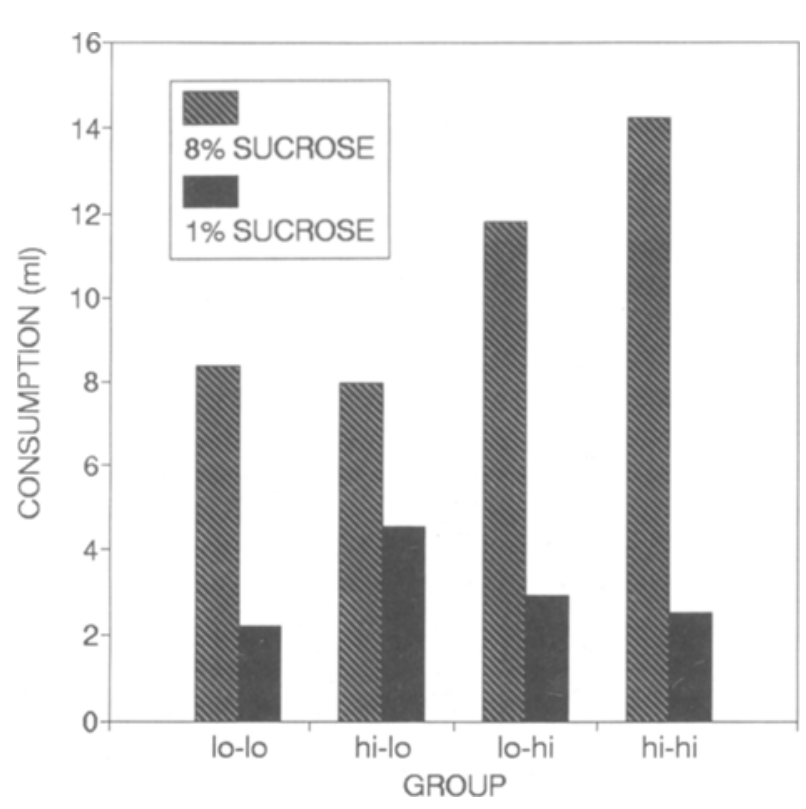

Figure 1. Mean milliliters consumed by each group over all of testing of the flavor that had been associated with $8 \%$ sucrose and the flavor that had been associated with 1\% sucrose in Experiment 1. Consumption was allowed to vary in training. High deprivation was a $14 \mathrm{~g}$ per day deprivation schedule; low deprivation was ad-lib access to chow.

the difference was smallest for Group hi-lo and largest for Group hi-hi.

Analysis of variance included training deprivation, testing deprivation, flavor (paired with $8 \%$ vs. paired with $1 \%$ ), solution containing flavors in test ( $1 \%$ vs. $8 \%$ sucrose), and days as factors. The difference due to flavor was significant $[F(1,37)=116.67]$, reflecting the preference for the flavor that had been paired with $8 \%$ sucrose. Rats also consumed more of both flavors if they had been trained under high deprivation $[F(1,37)=7.20]$ and if they were tested under high deprivation $[F(1,37)=$ 24.62]. The difference due to flavor was larger when testing was conducted under high deprivation [flavor $\times$ testing deprivation, $F(1,37)=15.01]$. The flavor $\times$ training deprivation interaction was not significant, but the flavor $\times$ testing deprivation $\times$ training deprivation interaction was significant $[F(1,37)=4.68]$. Subsequent Newman-Keuls tests showed that the amount consumed in testing of the flavor that had been paired with $8 \%$ sucrose was significantly greater than the amount consumed of the flavor that had been paired with $1 \%$ sucrose for every group except Group hi-lo.

The preference for the flavor associated with $8 \%$ sucrose over the flavor associated with $1 \%$ sucrose was larger when the test solution was $8 \%$ sucrose rather than $1 \%$ sucrose $[F(1,37)=5.17]$, but the relationship among groups did not depend on test solution (all $F \mathrm{~s}<1$ ).

Rats consumed more on Test Day 2 than on Test Day 1 $[F(1,37)=4.99]$, and the preference for the flavor paired with $8 \%$ sucrose was larger on Test Day 2 than on Test Day $1[F(1,37)=8.67]$. The interaction of days, preference, training deprivation, and testing deprivation was also significant $[F(1,37)=4.97]$. This interaction occurred because preference for the flavor paired with $8 \%$ sucrose increased from Day 1 to Day 2 of testing for all groups except Group lo-lo.

\section{Discussion}

In training in Experiment 1, rats consumed more 8\% sucrose than $1 \%$ sucrose, and rats trained under high deprivation consumed more of both concentrations of sucrose than those trained under low deprivation. Furthermore, in training the difference in consumption between the two sucrose concentrations was larger for rats trained under high deprivation. Results in testing almost mirrored these consumption differences in training. In testing, rats drank more of the flavor that had been associated with $8 \%$ sucrose than of the flavor that had been associated with $1 \%$ sucrose. They also drank more in test if they had been trained under high deprivation rather than low (and of course also drank more in test if they were tested under high deprivation rather than low). Although the difference in consumption between the two flavors in testing was not larger for rats that had been trained under high deprivation, there was a significant training deprivation $x$ testing deprivation $\times$ flavor interaction in testing. This interaction reflected the fact that the largest conditioned flavor preference occurred for Group hi-hi, and no preference occurred at all for Group hi-lo.

Fedorchak and Bolles (1987) found that preference for a flavor paired with sucrose over a flavor paired with water was enhanced by increasing deprivation in test. Experiment 1 extends this result to a preference based on sucrose concentration and to a different set of procedures. Fedorchak and Bolles gave flavors for $24 \mathrm{~h}$ in training with no concurrent access to unflavored water, used a different deprivation procedure from ours, and gave rats a 4-h, two-bottle test between flavors. Thus the finding that increased deprivation in testing facilitates a conditioned preference based on sucrose generalizes across a considerable difference in procedures. The effects of test deprivation on the conditioned flavor preference were larger than the effects of training deprivation, indicated by the failure of Group hi-lo to show a conditioned flavor preference.

Deprivation effects on the preference for the flavor paired with $8 \%$ sucrose were the same whether the test solution was $8 \%$ sucrose or $1 \%$ sucrose. This rules out an interpretation of the present results in terms of an effect of deprivation on generalization to novel flavorsolution combinations. The reasoning is as follows. When the test solution is $8 \%$ sucrose, the new flavor-solution combination is the combination of the flavor paired in training with $1 \%$ sucrose now given in $8 \%$ sucrose. In the test, this was the less preferred flavor. In this case, if a group consumed more of the novel flavor-solution combination, less of a consumption difference favoring 
the flavor associated with $8 \%$ sucrose would be shown. When the test solution is $1 \%$ sucrose, the new flavorsolution combination is the combination of the flavor that was paired in training with $8 \%$ sucrose now given in $1 \%$ sucrose. In this case, if a group consumed more of the novel flavor-solution combination, more of a consumption difference favoring the flavor associated with $8 \%$ sucrose would be shown. Thus, if generalization to new flavor-solution combinations is facilitated by training under high deprivation, the effect of training deprivation on preference for a flavor paired with $8 \%$ sucrose should have been larger when flavors were presented for testing in $1 \%$ sucrose. This did not occur, suggesting that an effect of deprivation on generalization to novel flavorsolution combinations was not responsible for these results.

\section{EXPERIMENT 2}

Experiment 2 was the same as Experiment 1, except that concentration of saccharin was varied instead of concentration of sucrose.

\section{Method}

The subjects were 42 rats of the same description as those employed in Experiment 1. There were 11 rats in Groups lo-lo and hi-lo and 10 rats in Groups hi-hi and lo-hi. The procedures were identical to those used in Experiment 1, except that the flavors were given in either $.15 \%$ saccharin or $.03 \%$ saccharin. Capaldi et al. (1983) used $.15 \%$ saccharin in their experiments.

\section{Results}

In training, high-deprivation rats consumed $6.9 \mathrm{ml}$ of $.15 \%$ saccharin and $1.5 \mathrm{ml}$ of $.03 \%$ saccharin. The comparable figures for the low-deprivation rats were $3.1 \mathrm{ml}$ and $2.1 \mathrm{ml}$. Analysis of variance showed that the difference due to deprivation was significant $[F(1,38)=10.24]$, as was the difference due to concentration of saccharin $[F(1,38)=102.26]$. The difference in consumption as a function of saccharin concentration was larger for rats trained under high deprivation $[F(1,38)=48.44]$.

Figure 2 shows the mean milliliters consumed of each flavor in testing for each group in Experiment 2. Figure 2 suggests that each group drank more of the flavor associated with the higher concentration of saccharin, and that this difference was larger for rats that had been trained under high deprivation than for rats trained under low deprivation. Analysis of variance including the same factors as in Experiment 1 showed that preference for the flavor associated with the higher concentration of saccharin was significant $[F(1,38)=32.54]$, and this difference was larger for rats that had been trained under high deprivation $[F(1,38)=4.82]$. No other differences were significant. Subsequent Newman-Keuls tests showed that consumption in testing of the flavor that had been paired with the higher concentration of saccharin was significantly greater than consumption of the flavor that had been paired with the lower concentration of saccharin for groups trained under high deprivation (hi-lo and hi-hi), but not for groups trained under low deprivation (lo-hi and lo-lo).

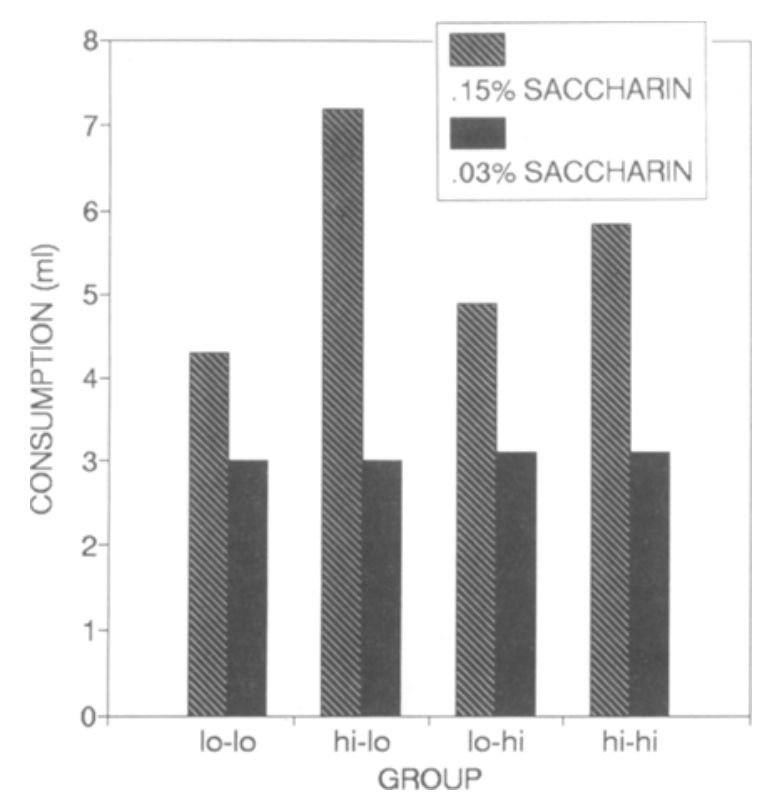

Figure 2. Mean milliliters consumed by each group over all of testing of the flavor that had been associated with $.15 \%$ saccharin and the flavor that had been associated with $.03 \%$ saccharin in Experiment 2. Consumption was allowed to vary in training. High deprivation was a $14 \mathrm{~g}$ per day deprivation schedule; low deprivation was ad-lib access to chow.

\section{Discussion}

Increasing deprivation in testing did not enhance the flavor preference that was based on saccharin concentration, which is consistent with Fedorchak and Bolles's (1987) results. They found that increased deprivation in testing did not enhance preference for a flavor paired with saccharin $(.028 \%$ or $.25 \%)$ over a flavor paired with water. Experiment 2 extends this finding to a preference based on saccharin concentration and to the use of different procedures. More importantly, Experiment 2 shows that training deprivation can have a different effect on conditioned flavor preferences than testing deprivation has. In Experiment 2, increased deprivation in training facilitated conditioned preferences that were based on saccharin concentration.

One obvious interpretation of how deprivation may influence the conditioning of flavor preferences is that it affects consumption. In both Experiments 1 and 2, the greater training consumption of the higher concentration was enhanced by higher deprivation in training. Perhaps effects of deprivation on learning of the preference in both Experiments 1 and 2 reflect these consumption differences. Pliner (1982), using human subjects, found that preference for unfamiliar juices increased with exposure, and Birch and Marlin (1982) obtained similar results with children. However, arguing against an interpretation of deprivation effects in terms of effects on consumption are results obtained by Holman (1975) using rats. Holman reported that there was no preference between two flavors of saccharin, one of which had been offered for $60 \mathrm{~min}$ and one for $5 \mathrm{~min}$. This different exposure length did pro- 
duce differences in amount consumed, but these differences did not produce a flavor preference. To our knowledge no one has investigated whether pairing different amounts of sucrose with flavors produces a conditioned flavor preference. Because differences in exposure could in theory account for the results of Experiments 1 and 2 , it is important to determine whether deprivation affects conditioned flavor preferences when consumption of the flavored solutions is equated.

In Experiments 1 and 2, the deprivation procedure differed from that used by Capaldi et al. (1983) and Capaldi et al. (1991). Capaldi and her colleagues used relatively high levels of deprivation. Recall that these experiments suggested that the reinforcing effect of sweetness is not increased by deprivation. Perhaps the reinforcing effect of sweetness is increased by deprivation when low deprivation consists of ad-lib access to food, but the reinforcing effect of sweetness does not continue to increase with further increases in deprivation and may even decrease. Experiments 3 and 4 equated consumption in training and used the deprivation procedure employed by Capaldi et al. (1983) and Capaldi et al. (1991). Experiment 3 examined flavor preferences based on concentration of sucrose; Experiment 4 did the same for saccharin.

\section{EXPERIMENT 3}

\section{Method}

Subjects. The subjects were 32 male albino rats 149 days old at the beginning of deprivation. They were 78 days old upon arrival from Holtzman (Madison, WI), and they had previously been run in a one-way escape-avoidance task. The rats were assigned to new groups balancing as evenly as possible for groups in the previous experiment. They had been fed $14 \mathrm{~g}$ of Wayne Lab Blox per day for 47 days and had food ad lib for 15 days prior to Day 1 of deprivation in this experiment.

Materials. The materials used were the same as in Experiment 1.

Procedure. All training and testing occurred in the rats' home cages. Water was available ad lib throughout the experiment in 350-ml brown bottles mounted on the right of the cage front. Food was removed on the day preceding Day 1 , and subsequently rats were fed $35 \mathrm{~g}$ of food (Wayne Lab Blox) on odd numbered days at 8:30 p.m. and nothing on even numbered days. This is the deprivation procedure used by Capaldi et al. (1983) and Capaldi et al. (1991). On this deprivation procedure, rats customarily consume the entire ration during the first night after it is received. Training began on Day 11. All training and testing began at 5:30 p.m.; therefore, low- and high-deprivation training occurred at 21 and $45 \mathrm{~h}$, respectively, after food was given.

During the first 4 training days, rats to be trained under high deprivation received $5 \mathrm{ml}$ of a solution on Days 11 and 13 (under high deprivation); rats to be trained under low deprivation received solutions on Days 12 and 14 (under low deprivation). The solutions remained on the cages until the next evening. Rats always consumed all the solutions. For half of each deprivation group, cinnamon was always paired with $8 \%$ sucrose and wintergreen was always paired with $1 \%$ sucrose; for the other half, the reverse was true. The order of presentation of the two flavors across these two training days was counterbalanced within each subgroup. The tubes were placed on the left side of the cage front so that the tip of the spout was about $2 \mathrm{~cm}$ from the cage floor and 4-5 cm from the left wall. The rats were trained on a total of 20 days, but only half the rats received trials on any particular day. High-deprivation rats were trained only on odd numbered days (high-deprivation days), and low-deprivation rats were trained only on even numbered days (low-deprivation days); so all rats received a total of 10 pairings of each flavor with the appropriate sucrose concentration. On a training day, both flavors were presented successively for $15 \mathrm{~min}$ each. No flavors or solutions were given rats on their nontraining days. The order of presentation of the two flavors was counterbalanced within each subgroup and alternated across presentations (i.e., cinnamon first and wintergreen second on one day, wintergreen first and cinnamon second on the next day, and so on for half the rats, and the reverse for the other half). The mean amounts of sucrose solution consumed over all training days for rats trained under high deprivation were $4.90 \mathrm{ml}$ of $8 \%$ sucrose and 4.89 of $1 \%$ sucrose; rats trained under low deprivation consumed $4.86 \mathrm{ml}$ of $8 \%$ sucrose and 4.90 of $1 \%$ sucrose. These differences between deprivation groups and sucrose concentrations were not significant $(F<1)$.

On each of 8 test days, the rats received two consecutive $10-\mathrm{min}$ two-bottle choice tests between $40 \mathrm{ml}$ of their training cues. Because tests were given every day, for each rat half the test days were under low deprivation and half were under high deprivation. The tests began at 5:30 p.m. and were spaced $30 \mathrm{~min}$ apart. Flavors were given in $8 \%$ sucrose on the 1st and 4th days of each 4-day sequence and in $1 \%$ sucrose on the 2 nd and 3 rd days for half the rats, and in the reverse sequence for the other half. The position of the cues on the first tests of the 1st and 4th days was cinnamon on the left and wintergreen on the right for half the rats in each subgroup; the flavors were in the opposite positions for the other rats. These initial positions were reversed for all rats on the 2 nd and 3rd days of each 4-day sequence. All rats began their second 10-min test with flavors in the opposite positions from those on the initial test.

\section{Results}

Figure 3 shows the mean amount consumed (in milliliters) in testing of the flavor paired with $8 \%$ sucrose and the flavor paired with $1 \%$ sucrose for each group. As can be seen in Figure 3, each group drank more of the flavor paired with $8 \%$ sucrose, and this difference was larger for rats trained under high deprivation than for rats trained under low deprivation. Analysis of variance included training deprivation, testing deprivation, test solution (8\% sucrose vs. $1 \%$ sucrose), flavor (paired with $8 \%$ vs. paired with $1 \%$ ), test (first vs. second), and days as factors. Consumption of the flavor that had been paired with $8 \%$ sucrose was significantly greater than consumption of the flavor that had been paired with $1 \%$ sucrose $[F(1,30)=$ 21.77], and this difference was larger for groups trained under high deprivation [flavor $\times$ training deprivation, $F(1,30)=7.15]$. This difference in consumption between the two flavors did not vary with deprivation in testing $(F<1)$, nor was the interaction of training deprivation, testing deprivation, and flavor significant $(F<1)$. Newman-Keuls tests showed that preference for the flavor paired with the higher concentration was significant for groups trained under high deprivation (Groups hi-hi and hi-lo), but not for groups trained under low deprivation (Groups lo-lo and lo-hi).

There were no significant interactions involving test solution. Rats consumed more on high-deprivation tests than on low-deprivation tests $[F(1,30)=10.54]$, and they con- 


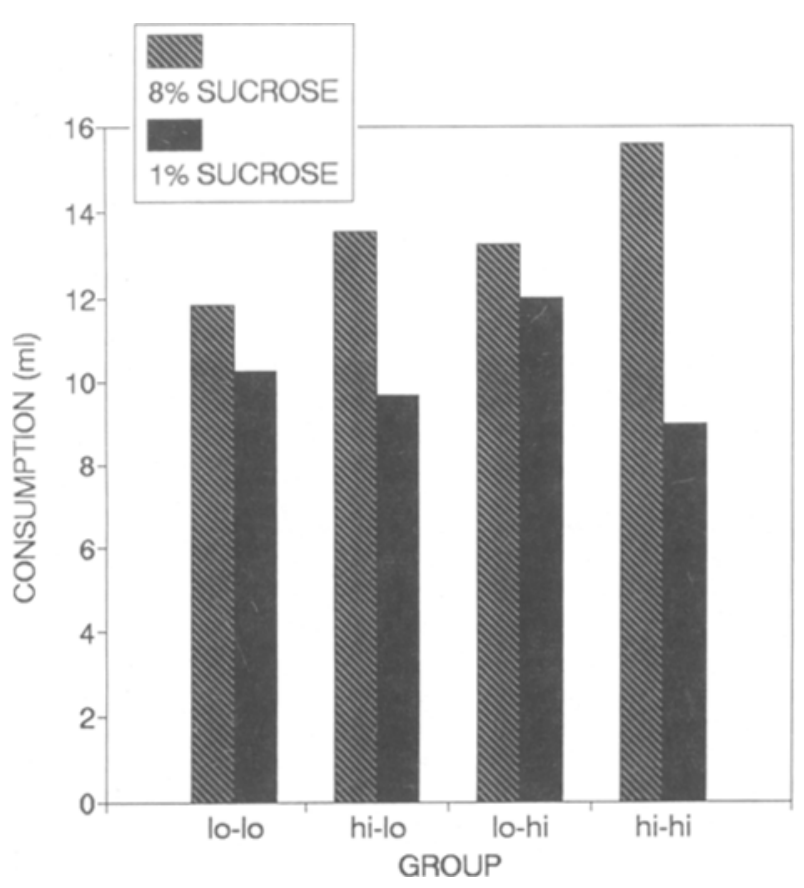

Figure 3. Mean milliliters consumed by each group over all of testing of the flavor that had been associated with $8 \%$ sucrose and flavor that had been associated with $1 \%$ sucrose in Experiment 3. Rats were given $5 \mathrm{ml}$ of each flavored solution each day in training. High deprivation was 45 h of deprivation; low deprivation was $21 \mathrm{~h}$ of deprivation.

sumed more when the test solution was $8 \%$ sucrose than when it was $1 \%$ sucrose $[F(1,30)=42.19]$. Flavor preferences were larger on the first test within the day $[F(1,30)=15.12]$ but did not change over days.

\section{Discussion}

Experiment 3 showed that the conditioned preference based on sucrose concentration was significantly larger for rats trained under high deprivation than for rats trained under low deprivation when the amount consumed in training was controlled and when relatively high deprivation levels were used. Indeed, rats trained under the lower deprivation in Experiment 3 failed to show a significant preference for the flavor paired with the higher sucrose concentration. In Experiment 1, in which the amount consumed in training varied with deprivation and in which lower deprivation levels were used, the conditioned flavor preference was significant for groups trained under low deprivation and for Group hi-hi. Thus, Experiment 3 showed a larger effect of training deprivation on the conditioned flavor preference than did Experiment 1, and a smaller effect of testing deprivation. The difference in the effect of training deprivation in the two experiments could have been due to either or both of the major procedural differences between the experiments. The difference in the effect of testing deprivation seems most likely to have been due to the different deprivation levels employed in the two experiments. Fedorchak and Bolles
(1987) found that increasing hunger in test enhanced a flavor preference based on sucrose when all rats had been trained under a common deprivation level and presumably had consumed the same amount of sucrose in training. The failure to find an effect of testing deprivation in Experiment 3 does not, then, seem attributable to our having equated consumption in training. A more likely hypothesis is that this result was due to the higher deprivation levels used in Experiment 3 than were used in Experiment 1 and in the Fedorchak and Bolles (1987) experiment.

\section{EXPERIMENT 4}

The design of Experiment 4 was identical to that of Experiment 3, except that saccharin was used rather than sucrose.

\section{Method}

Subjects. The subjects were 20 naive male albino rats, 100 days old at the beginning of the experiment. They were 75 days old upon arrival at the laboratory from Holtzman (Madison, WI).

Materials and Procedure. The procedure was the same as that in Experiment 3, except that training and testing began at 8 a.m., and feeding occurred at 4:30 p.m. Thus, low deprivation occurred at $15.5 \mathrm{~h}$ after food was given and high deprivation occurred at $39.5 \mathrm{~h}$. Flavors were delivered in $5 \mathrm{ml}$ of .15\% saccharin or $.03 \%$ saccharin. Rats consumed all the solutions in training.

\section{Results}

Figure 4 shows the mean milliliters consumed of the flavors that had been paired with $.15 \%$ saccharin and with $.03 \%$ saccharin for each group. As can be seen in Figure 4, each group preferred the flavor that had been paired with $.15 \%$ saccharin, and this difference was largest for Group hi-hi. Analysis of variance including the same factors as in Experiment 3 showed that the preference for the flavor associated with .15\% saccharin was significant $[F(1,18)=25.38]$. This preference did not interact with training deprivation $(F<1)$, or testing deprivation $[F<1)$, but the triple interaction, flavor $\times$ training deprivation $\times$ testing deprivation was significant $[F(1,18)=$ 5.81]. Examination of Figure 4 shows that rats tested under the same deprivation level as that experienced in training (Groups hi-hi and lo-lo) showed a larger preference than did rats tested under a different deprivation level (Groups hi-lo and lo-hi). Subsequent Newman-Keuls tests showed that the difference in consumption between the two flavors in testing was significant for each group. Rats consumed more on the high-deprivation tests than on the low-deprivation tests $[F(1,18)=45.55]$ and consumed more when the test solution was .15\% saccharin than when it was $.03 \%$ saccharin $[F(1,38)=5.17]$. There were no significant interactions involving test solution; flavor preferences were the same on each of the two tests within a day and did not change over days.

\section{Discussion}

When the amount consumed in training was equated and relatively high deprivation levels were used, deprivation 


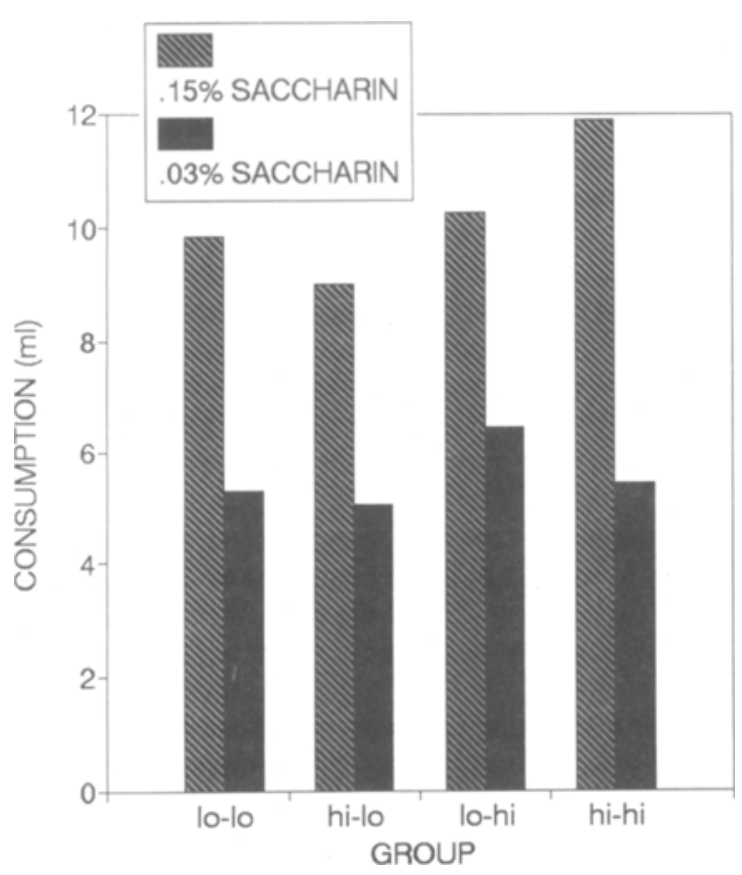

Figure 4. Mean milliliters consumed by each group over all of testing of the flavor that had been associated with $.15 \%$ saccharin and the flavor that had been associated with $.03 \%$ saccharin in Experiment 4. Rats were given $5 \mathrm{ml}$ of each flavored solution each day in training. High deprivation was $39.5 \mathrm{~h}$ of deprivation; low deprivation was $15.5 \mathrm{~h}$ of deprivation.

did not affect conditioning or expression of conditioned flavor preferences based on saccharin concentration. All groups showed a significant preference for the flavor paired with the higher concentration of saccharin. Changing deprivation from training to test reduced the size of the conditioned flavor preference, suggesting that deprivation stimuli had gained some control over the preference.

\section{GENERAL DISCUSSION}

Results with saccharin and sucrose were similar in some ways in these experiments. With both saccharin and sucrose, there was a conditioned preference for the flavor paired with the higher concentration. Also with both saccharin and sucrose, rats consumed more of both concentrations in training if trained under high deprivation. And following training with either saccharin or sucrose, total consumption in test was greater when testing occurred under high rather than low deprivation. Finally for both saccharin and sucrose, when consumption was allowed to vary in training, the difference in consumption between the high and low concentrations was greater for rats trained under high deprivation and this difference in consumption carried over into preference testing.

However, the effects of deprivation on conditioning of preferences were different for saccharin and sucrose.
There was a significant effect of training deprivation on the conditioned preference based on saccharin concentration in Experiment 2 when consumption varied and when relatively low deprivation levels were used. However, when consumption was equated between high- and lowdeprivation groups in training and higher deprivation levels were used, the effect of training deprivation on the saccharin-based preference disappeared. That is, the reinforcing value of saccharin concentration was not affected by deprivation when consumption was equated and relatively high deprivation levels were used. In contrast, the preference based on sucrose concentration was affected by deprivation even when consumption was equated and higher deprivation levels were used. Thus, the effect of training deprivation on sucrose-based preferences is not due to an effect of deprivation on consumption.

There were differences other than the use of sucrose or saccharin between Experiments 3 and 4 . Although the difference in deprivation levels was the same in these two experiments, the absolute levels of deprivation were slightly higher in Experiment 4 than in Experiment 3. Experiments 1 and 2, in which still lower deprivation levels were used, revealed that deprivation level in training affected strength of flavor preferences based on either saccharin or sucrose. It seems unlikely, although of course not impossible, that increasing deprivation levels produced an effect on conditioned flavor preferences in Experiment 3, and increasing them yet more prevented an effect in Experiment 4. There were also other minor procedural differences between Experiments 3 and 4 . The rats in Experiment 4 were naive, whereas the rats in Experiment 3 had been used in a previous experiment and thus had been on the deprivation schedule longer and were older. The rats in Experiment 3 were run at a different time of day than were those in Experiment 4, although all were run during their light cycle. Any of these procedural differences between Experiments 3 and 4 could have influenced the conditioned preferences obtained in Experiments 3 and 4.

Because there was no effect of deprivation on conditioned preferences in Experiment 4, the main effect of deprivation does not appear to be on the reinforcing effect of sweetness per se. If deprivation affected the reinforcing effect of sweetness, both saccharin- and sucrosebased preferences should have been affected by training deprivation.

At least three differences between sucrose and saccharin could have played a role in the different effects of deprivation on flavor conditioning with sucrose and saccharin. One important difference between sucrose and saccharin is that sucrose contains calories and saccharin does not. Fedorchak and Bolles (1987) suggested that increased deprivation in testing enhances expression of flavor preferences based on calories but not those based on flavor. Perhaps Fedorchak and Bolles's idea should be expanded to say that increased deprivation in training enhances learning of flavor preferences based on calories but not those based on flavor. This hypothesis would ex- 
plain why deprivation facilitated learning of flavor preferences based on sucrose and not those based on saccharin when exposure to the solutions under the two deprivation conditions was equated. However, it does not appear that deprivation enhances learning about calories per se. Fedorchak and Bolles found that preference for a flavor that had been paired with ethanol over a flavor that had been paired with water was not affected by training deprivation. Perhaps deprivation would affect conditioned preferences based on ethanol if the higher deprivation levels used here were employed. Or perhaps deprivation only affects learning about calories that accompany sweetness. Although this is possible, an alternative hypothesis that also rests on the calorie difference between sucrose and saccharin is the notion that sweetness is more reinforcing with increased deprivation only if increased sweetness is correlated with increased calories. This would explain why deprivation facilitates learning of flavor preferences based on sucrose concentration, but not those based on saccharin concentration or ethanol. This hypothesis is also compatible with Capaldi et al.'s (1991) results, which show that rats preferred a flavor associated with low deprivation when sweetened foods were used. In Capaldi et al.'s (1991) experiments, calories were not differentially associated with sweetness or flavors. Thus, those experiments measured the reinforcing effect of sweetness independently of associations of sweetness with calories.

Second, sucrose and saccharin differ in taste. Saccharin appears to have a dual taste to rats, both sweet and bitter (Morrison \& Jessup, 1977), but sucrose does not involve a bitter taste. Deprivation may affect the reinforcing effect of sweetness only when a relatively pure sweet taste is involved.

Third, different concentrations of sucrose and saccharin were used in these experiments. The higher concentration of saccharin used here is near the top of the preference gradient in the rat, whereas the higher concentration of sucrose used was lower than the most preferred concentration of sucrose. These concentrations were chosen because they were those used in the experiments of Capaldi et al. (1983) and Capaldi et al. (1991). In Experiment 4 here, all groups showed a significant preference for the flavor paired with the higher concentration of saccharin, whereas in Experiment 3, groups trained under the lower deprivation failed to show a significant preference for the flavor paired with the higher concentration of sucrose. It might be argued that the deprivation difference in Experiment 3 would not have occurred if a wider range of sucrose concentrations had been used. Arguing against this interpretation is the fact that the two concentrations of sucrose used here produced a greater difference in consumption in Experiment 1 than the two concentrations of saccharin used here produced in Experiment 2 . This suggests that the difference in reinforcing value between the two sucrose concentrations was if anything greater than that between the two saccharin concentrations.

Deprivation did affect conditioning of flavor preferences based on either saccharin or sucrose when consumption was allowed to vary freely in training. Many previous experiments have allowed consumption to vary freely. Capaldi (1991), for example, found that preference for a flavor that was associated with more sweetness and fewer calories than was another flavor was enhanced by increased deprivation in training. Rats could consume solutions freely in training in Capaldi's experiments, and test consumptions were highly correlated with training consumptions. Thus Capaldi's results may have been produced by consumption differences in training. Amount consumed in training in flavor preference conditioning experiments is not always reported (see, e.g., Fedorchak \& Bolles, 1987), making it difficult to know to what extent this variable might have influenced previous results.

\section{REFERENCES}

BIRCH, L. L., MARLIN, D. W. (1982). I don't like it, I never tried it: Effects of exposure on two-year-old children's food preferences. Appetite, 3, 353-360.

Bolles, R. C. (1972). Reinforcement, expectancy, and learning. Psychological Review, 79, 394-409.

CAPALDI, E. D. (1991). Hunger and the learning of flavor preferences. In E. D. Capaldi \& T. L. Powley (Eds.), Taste, experience, and feeding (pp. 157-169). Washington, DC: American Psychological Association.

CAPALDI, E. D., \& MYERs, D. E. (1982). Taste preferences as a function of food deprivation during original taste exposure. Animal Learning \& Behavior, 10, 211-219.

Capaldi, E. D., Myers, D. E., Campbell, D. H., \& Shefrer, J. D. (1983). Conditioned flavor preferences based on hunger level during original flavor exposure. Animal Learning \& Behavior, 11, 107-115.

CaPaldi, E. D., Sheffer, J., \& OWEns, J. (1991). Food deprivation and conditioned flavor preferences based on sweetened and unsweetened foods. Animal Learning \& Behavior, 19, 361-368.

Davis, J. D., \& Levine, M. W. (1977). A model for the control of ingestion. Psychological Review, 84, 379-412.

Fedorchak, P. M., Bolles, R. C. (1987). Hunger enhances the expression of calorie- but not taste-mediated conditioned flavor preferences. Journal of Experimental Psychology: Animal Behavior Processes, 13, 73-79.

Holman, E. W. (1975). Immediate and delayed reinforcers for flavor preferences in rats. Learning \& Motivation, 6, 91-100.

MORRISON, G. R., \& JessuP, A. (1977). Does saccharin have a dual taste for the rat? In J. M. Weiffenbach (Ed.), Taste and development: The genesis of sweet preference (pp. 42-47). Washington, DC: U.S. Government Printing Office.

Pliner, P. (1982). The effects of mere exposure on liking for edible substances. Appetite, 3, 283-290.

REvuSKY, S. H. (1967). Hunger level during food consumption: Effects on subsequent preference. Psychonomic Science, 7, 109-110.

SmIth, M., \& DUFFY, M. (1957). Consumption of sucrose and saccharin by hungry and satiated rats. Journal of Comparative \& Physiological Psychology, 50, 65-69.

(Manuscript received January 4, 1993; revision accepted for publication July $21,1993$. 